\title{
Pioneering Informed Consent for Return of Research Results to Breast Cancer Patients Facing Barriers to Implementation of Genomic Medicine: The Kenyan BRCA1/2 Testing Experience Using Whole Exome Sequencing
}

\author{
Rispah Torrorey-Sawe ${ }^{1,2 *}$, Nicole van der Merwe ${ }^{1}$, Simeon Kipkoech Mining ${ }^{2}$ and \\ Maritha J. Kotze ${ }^{1,3}$ \\ 1 Division of Chemical Pathology, Department of Pathology, Faculty of Medicine and Health Sciences, Stellenbosch \\ University, Tygerberg, South Africa, ${ }^{2}$ Department of Immunology, School of Medicine, College of Health Sciences, Moi \\ University, Eldoret, Kenya, ${ }^{3}$ National Health Laboratory Service, Tygerberg Hospital, Cape Town, South Africa
}

OPEN ACCESS

Edited by:

Yann Joly,

McGill University, Canada

Reviewed by:

Orland Diez,

Vall d'Hebron University Hospital,

Spain

Mohamed M. Abdel-Daim

Suez Canal University, Egypt

${ }^{*}$ Correspondence:

Rispah Torrorey-Sawe

torrorey@gmail.com;

rtorrorey@mu.ac.ke

Specialty section:

This article was submitted to

ELSI in Science and Genetics,

a section of the journal

Frontiers in Genetics

Received: 10 September 2019 Accepted: 12 February 2020

Published: 06 March 2020

Citation:

Torrorey-Sawe R,

van der Merwe N, Mining SK and Kotze MJ (2020) Pioneering Informed

Consent for Return of Research Results to Breast Cancer Patients

Facing Barriers to Implementation of

Genomic Medicine: The Kenyan

BRCA1/2 Testing Experience Using

Whole Exome Sequencing.

Front. Genet. 11:170.

doi: 10.3389/fgene.2020.00170
Introduction: Obtaining informed consent from study participants and disseminating the findings responsibly is a key principle required for ethically conducted clinical and genetic research. Reports from African researchers providing feedback on insights gained during the return of whole exome sequencing (WES) results to breast cancer patients treated in resource-limited settings is lacking.

Aim: The empirical process used to fill this gap in relation to BRCA1/2 variant detection using WES provided unique insights incorporated into a pathology-supported genetic testing algorithm for return of research results to Kenyan breast cancer patients.

Methods: The Informed consent form approved by the Moi Teaching and Referral Hospital in Kenya was adopted from a translational research study conducted in South Africa. Initially, the informed consent process was piloted in 16 Kenyan female patients referred for breast surgery, following a community-based awareness campaign. A total of 95 female and two male breast cancer patients were enrolled in the study from 2013 to 2016. Immunohistochemistry (IHC) results of estrogen receptor (ER), progesterone receptor (PR) and human epidermal growth factor receptor-2 (HER2) status were obtained from hospital records. DNA of patients with a family history of cancer was extracted from saliva and screened for pathogenic variants in the BRCA1/2 genes as the first step using WES.

Results: Ten patients approached for participation in this study declined to sign the informed consent form. Data on $\mathrm{IHC}$ used as a proxy for molecular subtype were available in 8 of 13 breast cancer patients (62\%) with a family history of cancer. Five BRCA1/2 variants of uncertain clinical significance were detected, as well as a pathogenic BRCA2 variant (c.5159C > A; S1720*) in a female patient eligible for return of WES results. 
Conclusion: Experience gained during the qualitative pilot phase was essential to overcome challenges associated with the translation of sophisticated genetic terms into native African languages. Detection of a pathogenic BRCA2 variant in a patient with familial breast cancer, frequently associated with hormone receptor-positive breast carcinoma as reported in this case, led to a high level of confidence on which to base risk management in future. Implementation of new technologies alongside standard pathology provides a practical approach to the application of genomic medicine in Africa.

Keywords: informed consent, genetics, genomics, pathology, return of results, Africa

\section{INTRODUCTION}

A gap in knowledge exists regarding the application of national and international ethical guidelines for research in Africa and other resource-poor settings (Alfano, 2013). Language and cultural barriers complicate the process of obtaining informed consent for genetic studies in rural African settings and data on solutions based on real-life experience are limited (Bhutta, 2004; de Vries et al., 2015; Adebamowo et al., 2018). In Kenya, obtaining informed consent for research and return of genetic results is a major challenge as the consent process is required to meet international standards, despite cultural diversity and lack of genetic counsellors or other healthcare professionals with experience in genomic research.

No standard format exists for obtaining informed consent from research participants applicable to both developed and developing countries. Careful planning is therefore required to ensure that ethical values are applied appropriately, especially in African countries where genomic research has not previously been performed (Afolabi et al., 2014; de Vries et al., 2015). Most emphasis appears to be placed on participant enrollment rather than ensuring a thorough understanding and comprehension of the project goals (Bhutta, 2004; Marshall, 2007; Dekking et al., 2014). A successful consenting process requires that potential study participants are brought to a high level of understanding how the purpose, methods, and risks of a study related to their personal or family medical conditions (Emanuel, 2000; Gupta et al., 2012). Good ethical conduct requires that the participant is allowed to make a voluntary and uncoerced decision whether or not to participate in a given study (Gupta et al., 2012). Therefore, only by obtaining true informed consent before the commencement of a study, can the standards for respect for people be achieved (Munung et al., 2016). Previous studies have shown that consenting illiterate individuals may be intimidating to them, which can further exacerbate challenges experienced during the consenting process (Alaei et al., 2013; Marshall et al., 2014). Even among literate patients, some may think that signing a consent form for research is part of their treatment regime (Bhutta, 2004). Therefore, managing participant expectations about feedback of research findings, either in a group context or on an individual basis, is an important part of the informed consent process.
Many approaches for return of research results have been reported. Knoppers et al. (2015) summarized these into four methods considered appropriate in the era of whole exome/genome sequencing. The first involves the use of filters, specific gene panels or targeted sequencing to reduce the potential for variants of uncertain clinical significance (VUS) and incidental findings. In the second approach, research results can only be returned when requirements for analytical validity, clinical significance and actionability (ACA) is met or the findings have personal utility and are clearly of essential relevance to health. The third criterion is a case-by-case approach where the communication of incidental findings obtained both in the research and clinical settings is evaluated on an individual basis. In a research setting, consulting a research ethics committee is required if the feedback plan was not included in the informed consent form (ICF). In the clinical setting, on the other hand, the treating clinician may consult with colleagues and depending on age, prognosis and other personal circumstances of the patient, findings that are outside of the primary indication of the test used in the research may be communicated to the patient (Jarvik et al., 2014). The noreturn fourth criterion applies when an individual's results from genome-based testing within the research context is used for the purpose of producing generalizable findings as opposed to demonstrating clinical utility. Research participants generally express great interest in receiving most classes of genetic findings, especially those with potential clinical significance (Christenhusz et al., 2013; Jarvik et al., 2014). Many research participants reportedly sought all of these results regardless of whether actionable, clinically significant or discovered incidentally (Wolf, 2013; Appelbaum et al., 2014). A multi-disciplinary team of knowledgeable health professionals with experience in genetic testing and interpretation skills are required to provide this kind of information.

Lack of genetic counseling services in Kenya, a country where breast cancer is the most common $(\sim 23 \%)$ form of cancer with approximately $75 \%$ of patients dying within 5 years of diagnosis (Ministry of Health Kenya, 2013), hampers the incorporation of genomics in clinical practice. Previous studies performed in western Kenya revealed early onset, aggressive breast cancer and lack of routine tumor subtyping to direct optimal treatment (Busakhala and Torrorey, 2012; Sawe et al., 2016, 2017). Development of screening models that can be 
translated into targeted risk reduction intervention strategies as the purpose of this study, is therefore needed to overcome language and other barriers to personalized genomic medicine in various cultural settings.

According to the Helsinki Declaration, it is an ethical obligation of researchers to make available or publish information about the outcome and results obtained in clinical research (World medical Association, 2013). In African genomic studies, provision for feedback of genetic results to individual study participants is absent in most ICFs used by investigators within the H3Africa (Human Heredity and Health in Africa) Consortium (Munung et al., 2016). de Vries et al. (2017) examined the existing ethics regulatory framework for genomic research and biobanking in 22 African countries including South Africa, and reported that only seven (Botswana, Cameroon, Ethiopia, Rwanda, Malawi, Sudan, and Uganda) specifically refer to the return of genetic results. A general concern is the impact of genetic research on family members, and whether or not these should also be included in the feedback. With regard to incidental research results, disclosure guidelines stipulate that (1) results should not be disclosed to relatives or third parties without written permission (Ethiopia), (2) disclosure should be determined by the investigator based on test sensitivity and specificity (Malawi), (3) participants' consent is required (Malawi, Botswana, and Cameroon) and mandatory involvement of a genetic counselor (Malawi and Botswana), (4) notification of test availability outside the research setting should carefully consider who has access to study results (Cameroon), and (5) policies for feedback and precautions should be in place to prevent unauthorized disclosure (Sudan). Uganda is the only country that stipulates that any results that are of clinical relevance, including incidental findings, must be returned to study participants. In Kenya no guidelines have been published for return of genomic research results, hence the current study adopted a research translation model developed in South Africa using a pathology-supported genetic testing (PSGT) strategy (Kotze, 2016).

Return of genetic results to study participants is challenged by the complex nature of integrating genetic findings into personal healthcare. A broad range of clinical information needs to be collected for genetic information to be meaningfully interpreted. Furthermore, to prevent unnecessary interventions, development and maintenance of secure open-access database resources are required to perform follow-up surveys and track long-term health outcomes of individual patients. For this purpose, substantial investment has been made in South Africa to develop the PSGT platform at the interface between the research laboratory and clinical practice. In the case of breast cancer, both germline and tumor genetics are taken into account to help guide treatment decisions across the continuum of care (Grant et al., 2013, 2019; Kotze et al., 2015; van der Merwe et al., 2017). The PSGT algorithm developed in South Africa was pioneered in Kenya to return actionable research results to patients with breast cancer. The ethical framework developed for this purpose provides a sound basis for clinical intervention during and beyond the course of a single research objective (Baatjes, 2018). The problems encountered and insights gained using an informed consent document that made provision for the return of genomic results, helped to inform the implementation of PSGT in Kenya on an individual basis.

\section{MATERIALS AND METHODS}

\section{Ethics Approval}

The Moi University-MTRH Institutional Ethics and Research Committee approved the study under project number 000655. Renewal of this project has been approved on an annual basis toward the return of research results from 2019.

\section{Study Design}

A mixed-methods approach employing both qualitative and quantitative data collection through an embedded sequential exploratory design was employed to report on the process used to obtain informed consent from 97 eligible breast cancer patients (Figure 1) and return of actionable research findings.

\section{Informed Consent Process}

The study population consisted of 97 patients with histologically confirmed breast cancer who attended the Moi Teaching and Referral Hospital (MTRH) for treatment between 2013 and 2016. The ICF used was adopted with permission from a South African genetic study that makes provision for feedback of research results to participants, based on the successful introduction of $B R C A 1 / 2$ mutation testing in private practice and at Tygerberg Academic Hospital (Kotze et al., 2004; Schoeman et al., 2013). It was explained to participants that genetic results interpreted in a clinical context may be made known in cases (1) with a definite risk for developing (a second) breast cancer, (2) with a predisposition or risk factor(s) that is treatable or avoidable e.g., by lifestyle or dietary modification, and/or (3) who may need genetic counseling. A material transfer agreement was signed between Moi University in Kenya and Stellenbosch University in South Africa and an import/export permit obtained for sample transport from Kenya to South Africa.

\section{Data Collection}

The consent form was piloted in 16 females who presented with breast lumps, to gauge its ability to clearly and sufficiently relay information regarding the design and goals of the study conducted at MTRH. Where necessary, an interpreter who could speak the patient's native language was engaged to help translate the information in the consent form. In order to determine whether the patients understood what was discussed during the informed consent process, they were asked to repeat in their own words what had been explained to them. A questionnaire was administered to obtain demographic characteristics, disease status, medication use, family history and lifestyle risk factors as previously described (Busakhala and Torrorey, 2012; Sawe et al., 2016).

Evaluation of the family history was performed using the breast cancer referral screening tool (B-RST ${ }^{\mathrm{TM}}$ ) described by Bellcross et al. (2009). This table was used to record both patient responses to family history questions and to assess 


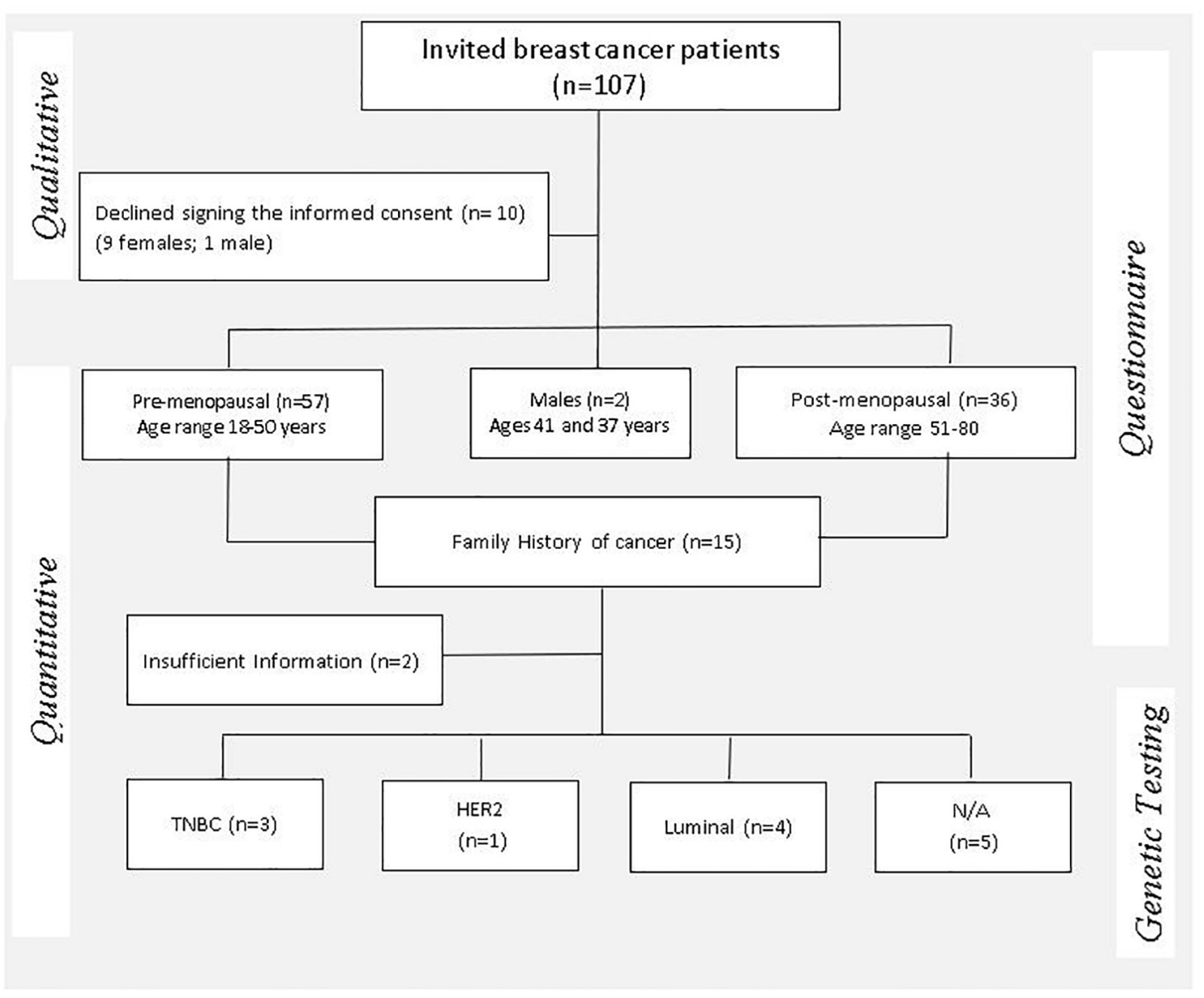

FIGURE 1 | Mixed methods study design flow chart illustrating the enrollment process of study participants and clinical indicators considered for eligibility of genetic testing. TNBC, triple-negative breast cancer; HER2, human epidermal growth factor receptor-2 positive; N/A, not available.

eligibility for genetic testing. Two or more checks in the table were regarded as positive, whereas the absence of family history or less than two checks were classified as negative. The selection of two parameters as the cut-off for a positive screen was adopted from published criteria used to define risk associated with highly penetrative, hereditary cancer syndromes (Bellcross et al., 2009).

\section{Immunohistochemistry}

Tumor histopathology was recorded and immunohistochemistry (IHC) of estrogen receptor (ER), progesterone receptor (PR), and human epidermal growth factor receptor-2 (HER2) determined as previously described (Sawe et al., 2016, 2017). Tissue samples were sectioned and fixed onto Flex IHC slides (Dako, Inc.), deparaffinized and hydrated, followed by antigen retrieval using the PT Linker system. The IHC staining was performed using a Dako Cytomation Autostainer Plus, followed by Hematoxylin nuclear counterstaining. For quality control purposes, known positive and negative control specimens were included for each antibody.

\section{Genetic Studies}

DNA was extracted from saliva using Oragene reagents (Ottawa, $\mathrm{ON}$, Canada), according to the instructions provided with this commercially available kit. All DNA preparation steps and analyses were performed at the Pathology Research and Central Analytical Facilities of Stellenbosch University by medical scientists registered with the Health Professions Council of South Africa (HPCSA). Given the lack of a regulatory framework for application of WES in Kenya, only pathogenic BRCA1/2 variants with well-established clinical guidelines were reported in this study, paving the way for extended WES data analysis and return of research results in Kenya.

Whole exome sequencing (WES) and mapping of sequenced data were performed as previously described by van der Merwe et al. (2017). The Ion AmpliSeq ${ }^{\text {rmTM }}$ Exome RDY Library Preparation protocol was used and template amplification was performed using the Ion $\mathrm{PI}^{\mathrm{TM}}$ Template OT2 200 Kit v3 (Thermo Fisher Scientific, Waltham, MA, United States). Semi-conductor sequencing on the Ion Proton system was performed using the Ion $\mathrm{PI}^{\mathrm{TM}}$ Sequencing 200 Kit v3 with the Ion PI ${ }^{\mathrm{TM}}$ Chip Kit v2. 
This method is designed to target all human exons, the coding regions of the human genome. A coverage depth of $15 \times$ was used for detection of potentially causative gene variants. In addition to the quality filters applied, rare variants were filtered on a population frequency of $<0.01 \%$ with a variant function set to exclude all synonymous variants.

Resulting variant call format (VCF) files were processed using the GeneTalk and wANNOVAR web-based tools for filtering and annotation of sequencing data. Annotated variants were downloaded and filtered for detection of variants in the BRCA1/2 genes associated with hereditary breast and ovarian cancer using a shell script. The script uses the grep command to search in the unfiltered VCF file for variants in the genes on the target gene list, which were sorted with Excel according to variant allele frequency and effect on the relevant amino acids. Bioinformatics tools freely available on the internet (Varsome ${ }^{1}$ and ClinVaR) ${ }^{2}$ were used for variant classification. These automatic variant classifiers evaluate the submitted variant according to the American College of Medical Genetics and Genomics (ACMG) guidelines (Kopanos et al., 2018). Each pathogenic criterion is weighted as very strong (PVS1), strong (PS1-4), moderate (PM1$6)$, or supporting (PP1-5), and each benign criterion is weighted as stand-alone (BA1), strong (BS1-4), or supporting (BP1-6) (Richards et al., 2015). Rare variants were verified with the Integrative Genomics Viewer, IGV 2.4 and Sanger sequencing used as the gold standard to confirm potential pathogenic variants detected in germline DNA.

\section{Return of Research Results}

Whole exome sequencing reports were generated for return of research results using the PSGT algorithm previously described by van der Merwe et al. (2017). This involves a questionnairebased assessment and evaluation of tumor molecular subtype as a pre-screen step for eligibility assessment and clinical interpretation of WES. PSGT was applied in this study to help distinguish between inherited breast cancer and patients with lifestyle-related risk factors associated with increased recurrence risk. Relevant data entered in the research database were extracted semi-automatically into adaptable reports using the Gknowmix $^{\text {TM }}$ research translation tool ${ }^{3}$ (Kotze et al., 2013). WES reports were authorized for disclosure of actionable results by HPCSA registered medical scientists as part of a multidisciplinary team responsible for the dissemination of genetic results. The data supporting the conclusions of this manuscript will be made available by the authors, without undue reservation, to any qualified researcher.

\section{RESULTS}

\section{Informed Consent}

One hundred and seven breast cancer patients were invited to participate in this study, of which 10 patients declined to

\footnotetext{
${ }^{1}$ https://varsome.com

${ }^{2}$ https://www.ncbi.nlm.nih.gov/clinvar

${ }^{3}$ https://www.gknowmix.org
}

provide consent for research (Figure 1). For assessment of family history across three generations, the B-RST ${ }^{\mathrm{TM}}$ family history tool was added to the consent form subsequent to a pilot phase conducted in 16 individuals. Between 30-60 min was spent with each patient to ensure that they understood the purpose of the study and why personal, family and lifestyle information was requested before a saliva sample was collected. The ICF was signed, and a saliva sample collected from 97 breast cancer patients after sufficient comprehension was demonstrated by each individual. Two patients requested that their saliva/DNA samples be destroyed at the completion of the study.

\section{Clinical Characteristics}

Table 1 summarizes the clinical characteristics of the study population, including 2 males and 95 females between the ages of 18 and 80 years, with a mean age of 46.9 years (SD 13.1). Family history of breast/ovarian cancer, male or bilateral breast cancer and triple-negative cancers were considered important clinical indicators for differential diagnosis of inherited and lifestylerelated breast cancer. Family history of cancer was reported in 13 female patients (13.5\%, aged 35-70 years). Four patients were below the age of 40 years; one had bilateral cancer; and three breast cancers were triple-negative according to $\mathrm{ER}, \mathrm{PR}$, and HER2 status The majority of study participants $(62.0 \%)$ were diagnosed with cancer before the age of 50 years, with a family history reported in seven $(54.0 \%)$ of these patients.

TABLE 1 | Clinical characteristics of 97 Kenyan breast cancer patients included in the study.

\begin{tabular}{|c|c|c|}
\hline Variables & Number & Percentage \\
\hline \multicolumn{3}{|c|}{ Age (years): Mean (SD) 46.9 (13.1) } \\
\hline \multicolumn{3}{|l|}{ Gender } \\
\hline Female & 95 & 97.9 \\
\hline Male & 2 & 2.1 \\
\hline \multicolumn{3}{|l|}{ Pathology } \\
\hline Adenocarcinoma & 1 & 1.0 \\
\hline Ductal cell carcinoma & 23 & 24.0 \\
\hline Infiltrating ductal carcinoma & 35 & 36.5 \\
\hline Lobular carcinoma & 2 & 2.1 \\
\hline Malignant phyloid tumor & 1 & 1.0 \\
\hline Mucinous & 3 & 3.1 \\
\hline \multicolumn{3}{|l|}{ Grade } \\
\hline I & 5 & 5.2 \\
\hline$\|$ & 22 & 22.9 \\
\hline III & 17 & 17.7 \\
\hline IV & 4 & 4.2 \\
\hline \multicolumn{3}{|l|}{ Breast cancer type } \\
\hline Luminal & 22 & 22.6 \\
\hline Triple negative & 8 & 8.2 \\
\hline HER2 & 4 & 4.2 \\
\hline Missing & 63 & 65.0 \\
\hline \multicolumn{3}{|l|}{ Family History } \\
\hline No & 84 & 86.6 \\
\hline Yes & 13 & 13.4 \\
\hline
\end{tabular}




\section{Genetic Results}

Table 2 summarized the tumor type, available IHC data and variants detected in the BRCA1 and BRCA2 genes in 13 patients with familial breast cancer. These include five variants of uncertain significance (VUS) in four patients, and a pathogenic variant c.5159C $>\mathrm{A}\left(\mathrm{S} 1720^{*}\right)$ in exon 11 of the BRCA2 gene. In the corresponding protein of the BRCA2 nonsense variant, codon 1720 is changed to a stop codon resulting in premature protein truncation. This variant was confirmed by Sanger sequencing (Figure 2).

\section{Return of Research Results}

The patient with the pathogenic BRCA2 mutation has been informed of this result by the treating clinician, with the assistance of the extended research team. The insights gained during the qualitative phase of the study involving the informed consent process was incorporated into the patient report used during the feedback process as reflected in Table 3. Return of research results according to the feedback plan in the ICF was facilitated by clinician support, taking patient perceptions and cultural barriers into consideration as well as analytical validation and clinical utility of the genetic findings.

\section{DISCUSSION}

This study addressed the lack of documentation of real-life experiences on the return of research results in rural African settings, where even basic IHC tumor subtyping was not routinely available to breast cancer patients at the time of recruitment. The finding that ER, PR and HER2 status was available in less than $50 \%$ of the study population highlights the potential for chemotherapy overtreatment in patients with early stage breast cancer and a missed opportunity to identify patients with triple-negative breast cancer linked to an increased risk of harboring pathogenic BRCA1 mutations (Chen et al., 2018). Since 2013, this research has evolved from pilot testing in 16 individuals to collection of saliva samples from 97 breast cancer patients and return of actionable research results to one of 13 cases with familial breast cancer. Whether to return individual research results to study participants remains an area of debate due to complexities associated with the potential reclassification of gene variants that may require a change in clinical management. However, non-disclosure of actionable findings may be unethical given the evolving responsibility of researches to recontact patients after reinterpretation of genomic research results (Bombard et al., 2019; Wong et al., 2019). Use of the PSGT algorithm incorporating the previous knowledge that pathogenic $B R C A 2$ variants are frequently associated with hormone-positive breast carcinoma increased our confidence level in return of WES-generated research results after confirmation by Sanger sequencing.

While the classification of BRCA2 c.5159C>A (S1720*) as a pathogenic variant is highly unlikely to change, the finding of VUSs in four Kenyan patients may require recontact of these patients in future in the event of reinterpretation, following family screening, functional studies or extended testing of

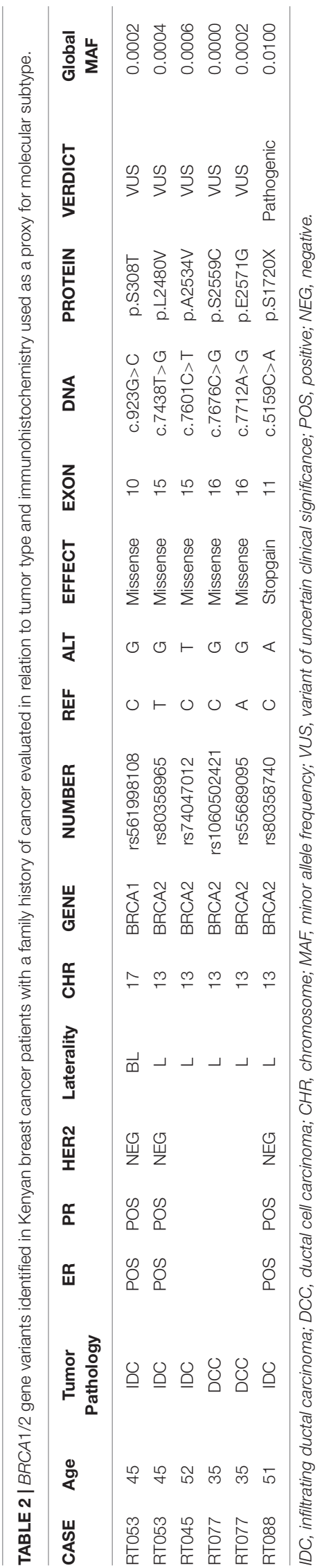



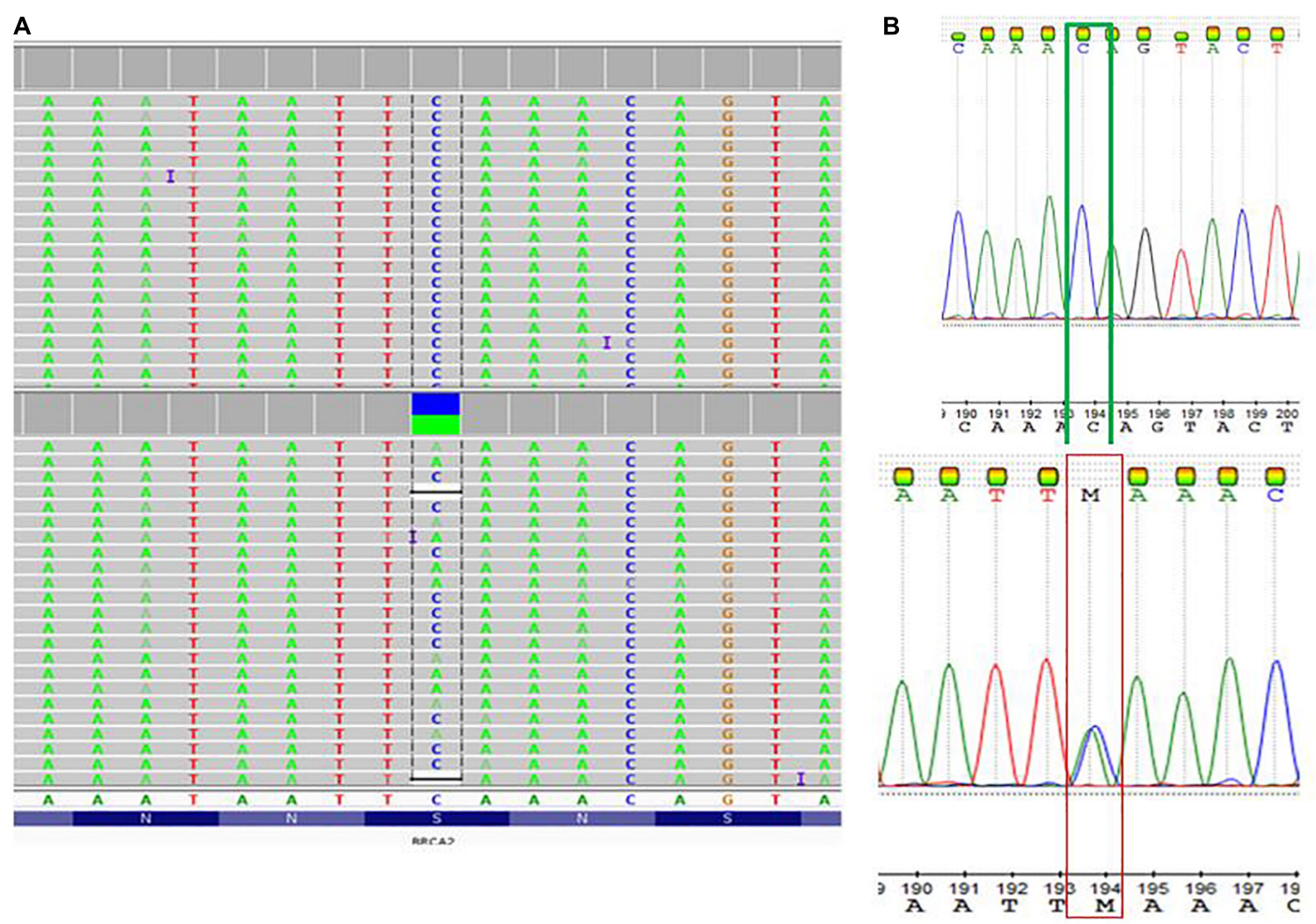

FIGURE 2 | Detection of a pathogenic BRCA2 variant C.5159C>A; S1720* (rs80358740; NM_000059.3.1). (A) Visualization of whole exome sequencing results using the Integrative Genome Viewer software tool. (B) The C to A base change at Genomic location 13: 32339514 (GRCh38) GRCh38 UCSC was confirmed by Sanger sequencing.

TABLE 3 | Framework for the return of research results based on the content of the informed consent form (ICF) and information included in the research database of 97 Kenyan breast cancer patients.

\section{Review of Informed consent form}

Eligibility assessment based on signed ICF that makes provision for return of research results.

Clinical relevance of genetic findings and option of genetic counseling a pre-requisite for return of research results.

Patient samples collected from 2013 before the requirement for researchers to recontact study participants in the event of variant reclassification that came into effect in 2019.

Investigators may be conflicted about returning research results, given the knowledge that genetics cannot fully account for phenotypic variability.

Approval for data sharing among genome researchers to gain collective knowledge from return of results and follow-up studies.

\section{Considerations before return of results}

Histopathology and immunohistochemistry results of breast carcinoma obtained from hospital records.

Analytical validation using Sanger sequencing as the gold standard confirmed the pathogenic BRCA2 variant detected by WES.

Variant reclassification is highly unlikely in the case of the pathogenic BRCA2 mutation identified by WES, while it may become necessary for a VUS in future.

Data on medication use and comorbidities captured in the research database are required for return of WES results relevant to breast cancer treatment.

Long-term participant engagement allows open communication with researchers aiming to close the gap between expectation and reality.

\section{Challenges addressed in report}

Maintaining confidentiality during the translation of data into the software program for the generation of an adaptable report.

$B R C A 2$ pathogenic variant in a patient eligible to obtain a report, but the same does not necessarily apply to at-risk family members. Updated WES report template includes a statement that further studies in an extended sample of breast cancer patients and family screening for the same variant may result in a variant reclassification.

Pathology-supported genetic testing facilitates evaluation of the clinical characteristics of each patient in relation to inherited-, lifestyle- or therapy-induced risk implications.

Availability of research translation resources crucial to cover the costs of validating WES results and contacting the participants for extended testing or report updates. additional unrelated breast cancer patients (Richards et al., 2015). Pathogenic BRCA2 variants are associated with an increased risk of other cancers, including ovarian and pancreatic cancer identified as an important consideration for surveillance in the Kenyan patient who was eligible for return of research results (Kopanos et al., 2018). Discussion of the uncertainties and 
potential for reclassification are therefore crucial to ensure that the outcome of genetic test results and impact on the extended family is understood by the treating clinician and patient.

The qualitative phase of this study was piloted in females referred to MTRH for a lumpectomy, following a breast cancer screening campaign in Western Kenya facilitated by the Academic Model Providing Access to Healthcare (AMPATH) community engagement initiative (Busakhala et al., 2016). Patients with benign breast lumps asked many questions related to a perceived risk that surgery may trigger cancer, with less emphasis on the reason for being approached by the investigator. This preparation phase for genetic analysis highlighted the importance of a well-designed ICF to provide patients with clear and adequate information regarding the study design and goals. The knowledge gained from the pilot phase was applied when obtaining informed consent from the 97 breast cancer patients finally enrolled for the quantitative phase of this study. An effort was made to ensure that participants understood how the study related to their breast cancer diagnosis, recurrence risk, and family members. Most of the breast cancer patients needed further clarification of medical terms used in the consent form, such as DNA and hormone replacement therapy. As previously highlighted by de Vries et al. (2015), these terms have no equivalent translation in the native African languages and therefore required extra time for an explanation in an attempt to improve comprehension.

A young patient hesitated to provide consent, but when probed as to why she was not willing to sign the form, she answered as follows; "I cannot read or write and I am ashamed of people to know about it." It has been reported that a major barrier to effective consenting is the level of literacy (Silverman et al., 2005). Although illiteracy is expected to be higher among elder people, it is now evident that even among the young in Kenya, it may still be a problem. Most illiterate individuals are ashamed of this fact and therefore refuse to accept or alternatively ask their next of kin to sign on their behalf. Considering that true informed consent is achieved only if the potential participant understands the purpose, methods, risks, and alternatives of a study in relation to his or her personal clinical context (Emanuel, 2000; Boga et al., 2011; Gupta et al., 2012; Tindana et al., 2012), illiteracy is likely to hinder this process. Similar to other studies, we used an interpreter familiar with the patient's dialect (Abeybsekera, 2018). A recommendation is that investigators in Kenya and other African countries embrace the utilization of tools that may be of value in low-literacy communities such as the use of picture files or speaking books. The latter resource employs cartoons in addition to text, which sounds when the corresponding button is pushed for a specific concept page. An ongoing pilot on the utility of these speaking books is expected to improve communication and may be available for widespread distribution and use across Africa ${ }^{4}$.

Muthoni and Miller (2010) reported that a diagnosis of breast cancer may lead to a state of low self-esteem, a feeling that may predispose to stigma and trauma and hence could deter both male and female patients from seeking early healthcare

${ }^{4}$ http://www.booksofhope.com/, n.d. advice. Moreover, some patients perceive a diagnosis of breast cancer as a death sentence and may delay treatment in order to protect their families from stigmatization. Rayne et al. (2016) reported a significant relationship between younger age of onset and fear of loss of hair or breasts in an urban population in South Africa. This may also be the case in Kenya, as ten patients who were approached in this study declined signing the ICF. Of these ten patients, one male displayed a "silent refusal attitude" at the beginning, which was characterized by hesitation to participate without openly refusing. Such behavior has previously been reported in a Kenyan study, which did not involve genetic investigations (Kamuya et al., 2015). Reasons for this attitude may be that participants do not want to offend the interviewer, especially after he/she spent their energy and time explaining about the study goals and expected outcomes in a manner that should clarify the difference between routine cancer treatment and potential feedback on genomic research results. Participants may avoid declining the request for voluntary research participation openly when the researcher is seen as part of the hospital setting to ensure continued benefit from the institution (Kamuya et al., 2014). Management of patient fears and expectations following breast cancer diagnosis may curb beneficial misconception in potential participants, while simultaneously ensuring true comprehension and voluntary participation in the research process. This is an important consideration given the reaction of a 20-year old female invited into the study, who ran away from the hospital ward due to fear of surgery. In South Africa, fear of genetic discrimination was identified as the major concern during the implementation of a $B R C A 1 / 2$ screening program more than 10 years ago (Kotze et al., 2004). This issue was addressed by weighing the benefits and risks associated with different types of genetic tests and correcting misconceptions in the context of breast cancer, where prophylactic surgery is an option. Knowledge about the role of reconstructive breast surgery may ease the fear in breast cancer patients and could guide risk reduction interventions accessible to Kenyan breast cancer patients.

As advocated by Akuoko et al. (2017), earlier presentation to the hospital could be achieved if healthcare providers, with support from the government, collaborate in developing approaches to improve the clinical outcome of patients with breast cancer. Disparities in genetic testing services available in high versus moderate/low-income countries such as Kenya (Hill et al., 2015), highlighted the need for financial assistance programs to remove economic barriers to breast cancer genetic testing (Jones et al., 2017). A systematic review of the costeffectiveness of healthcare programs involving genetic testing of the two major breast cancer genes, $B R C A 1$ and $B R C A 2$ indicated that family history-based screening programs are cost-effective (D'Andrea et al., 2016). Use of data integration tools such as PSGT incorporating WES to identify individuals at increased risk of inherited versus lifestyle-related forms of cancer or treatment-related co-morbidities, may further improve the costeffectiveness of testing.

More extended data analysis of WES reads beyond BRCA1/2 is an important consideration in genetically uncharacterized patients, especially those experiencing medication side effects or 
treatment failure. The potential value of simultaneous detection of pharmacogenetic markers relevant to tamoxifen resistance (Baatjes et al., 2017) has been mentioned in the feedback report of the pathogenic BRCA2 variant in one of the Kenyan patients studied. Results from such studies may inform and guide government policy toward the provision of medical coverage for genetic tests to improve patient outcome. The emerging duty to return genetic information is based on the principles of autonomy, beneficence, and the acknowledgment that translational genomic research cannot progress without the engagement of research participants (Lolkema et al., 2013; Bombard et al., 2019). Lack of genetic counselors in Kenya necessitates the development of an innovative approach toward breast cancer genetic testing and return of research results. Incorporating cognitive strategies through a multimedia approach may in future enhance comprehension of research procedures, instead of using a single face-to-face method when consenting and counseling patients in biomedical research (Antal et al., 2017).

The ICF used in this study clearly indicated that research results may be reported based on clinical relevance and/or the need for genetic counseling that may include support to at-risk family members of study participants. In this context, different models ranging from specific to tiered and broad consent (Munung et al., 2016) were evaluated by Van Der Merwe (2018), who conducted a qualitative study exploring South African stakeholder views on the return of individual research results and incidental findings. Stakeholders including clinicians, genetic counselors and medical scientists contended on the one extreme that broad consent would enable more information to be gathered for future use in research, and that the broad categories of potential harm, potential benefit and limitations should be covered - not necessarily all the details inbetween. On the other extreme, some stakeholders advocated for use of tiered consent that allows participants to break down the various aspects of consenting as far as they wish. Explanation of the return-of-results plan to every potential participant prior to study enrollment facilitated disclosure of actionable BRCA2 results in this study, which may in future be extended to pharmacogenetic WES data analysis using a clinical pipeline applicable to PSGT tailored to the needs of the individual in an adaptable report (Baatjes et al., 2017). The WES report that was generated for the Kenyan breast cancer patient with a pathogenic $B R C A 2$ variant was used by the treating clinician to disclose the results based on the steps stipulated in Figure 3. Considering that there is no standardized method for returning research results in Kenya, we applied the Clinical Sequencing Exploratory Research (CSER) Consortium and the Electronic Medical Records and

STEP 1: Review informed consent form (ICF)

Patient signed the ICF and selected sample storage with the option for return of research results:

ERt, PRt, HER2- breast cancer diagnosed in a Kenyan patient with a family history of cancer.

r.

STEP 2: Confirmation of WES data

Designed primers flanking the BRCA2 variant c.5159C $>$ A, p. Ser1720Ter (NM_000059.3.1):

WES result confirmed by Sanger sequencing used as the gold standard for analyticalvalidation.

STEP 3: Interpretation of genetic results in the context of clinical information available in the research database

The pathogenic BRCA2 variant ( 1580358740 ) is consistent with the Hereditary Breast and Ovarian Cancer syndrome:

All the 1st-degree family members (parents, siblings and children) have a $50 \%$ risk of carrying the mutation and are at increased risk of developing breast, ovarian and other cancers. Due to the familial implications, and with the consent of the patient, this information may be shared with family members.

STEP 4: Follow-up studies and clinical management of familial risk

The patient agreed to disclose the results to her children:

Patient was encouraged to re-contact the institution every few years to review/update family history and address any new issues that may be relevant to the family. Genetic counselling/consultation for predictive testing of at-risk relatives to facilitate cancer prevention is possible since the family mutation has been identified in a diagnostic setting and confirmed by Sanger sequencing.

STEP 5: Document feedback from the treating clinician on the clinical utility of WES
Treating clinician recommended that a copy of the report be kept in the file of the patient for future reference:
Expressed interest in pharmacogenetics related to tamoxifen resistance and made a note in the patient file to bring the
BRCA2 mutation-positive results to the attention of any clinician who may treat the patient in future.

FIGURE 3 | Five-step process used to disclose actionable research results to a study participant with BRCA2 pathogenic variant and documenting feedback from the treating clinician to determine clinical utility. 
Genomics (eMERGE) Network guidelines (Jarvik et al., 2014) based on the ACA (Analytical validity; Clinical significance and Actionability) criteria (Knoppers et al., 2015).

During result disclosure the treating clinician used a language that the patient is comfortable with and informed her about the risks and benefits of BRCA1/2 gene screening, as explained in the WES report. After gauging her feelings on receiving the genetic results and assessment of the level of understanding before proceeding to deliver the result, the patient confirmed that she still wants to know the outcome (test voluntariness) (Isles, 2013). Our experience in overcoming barriers to the disclosure process due to lack of standardized protocols or consensus guidelines on how and when to return genetic test results, contributed to the development of a framework for tiered informed consent applicable to research in Africa (Nembaware et al., 2019). Although there is a lack of genetic counselors in Kenya, this did not preclude disclosure of the genetic results. Effective feedback was achieved through joint consultation with the researcher who obtained the initial informed consent from the patient and clinicians involved in the genomic research project and treatment of the patient. Since adhering to the gold standard of involving genetic counselors or medical geneticists may not be practical in a resource-limited setting, primary care physicians and primary care nurses need to be empowered by education in this context (Van Der Merwe, 2018).

The results presented in this study are supported by many strengths, such as the inclusion of a qualitative research component that prepared us for potential problems during information transfer in the subsequent study. The initial reactions of Kenyan patients highlighted the needs of the study population during implementation of a genetic screening program, which in turn guided the way in which we approached the informed consent process and disclosure of genetic results. The cultural level and degree of diversity among patients were not assessed and could have affected their reactions and understanding of the goals of the project and hence acceptance to participate in the study.

\section{CONCLUSION}

This study describes the use of WES to screen for pathogenic $B R C A 1 / 2$ variants alongside clinico-pathological assessments used for interpretation of the findings obtained in Kenyan breast cancer patients. The challenges encountered during the informed consent process were contextualized into a framework for return of research results to patients with familial breast cancer. The pre-test process pioneered during this investigation identified problem areas addressed by the development of an adaptable report that enables disclosure of WES results in five steps. Detection of a pathogenic BRCA2 mutation (c.5159C>A; $\left.S 1720^{*}\right)$, in $1 / 13(8 \%)$ of the breast cancer patients with familial breast cancer confirmed that family history is an important indicator for the diagnosis of inherited breast cancer in Kenya. Novel insights gained as a result of this experience support the incorporation of new technologies integrated with standard pathology to facilitate the return of research results in low and middle-income settings, where challenges associated with the translation of sophisticated genetic terms into native African languages persist. As genetic research gains momentum on the African continent, innovative strategies such as PSGT combining pathology and genetic tests will become increasingly important to translate research into clinical practice. This is the first study performed in Kenya to determine the cause of familial breast cancer in patients using WES, which allows for extended data analysis beyond $B R C A 1 / 2$ at no additional cost in patients who experience treatment failure or medication side effects.

\section{DISCLOSURE}

MK is a director and shareholder of Gknowmix (Pty) Ltd., that developed a database tool for research translation under the auspices of the South African Medical Research Council.

\section{DATA AVAILABILITY STATEMENT}

The raw data supporting the conclusions of this article will be made available by the authors, without undue reservation, to any qualified researcher.

\section{ETHICS STATEMENT}

The studies involving human participants were reviewed and approved by the Institutional Review and Ethics Committee (IREC). Moi Teaching and Referral Hospital, Eldoret, Kenya. The patients/participants provided their written informed consent to participate in this study.

\section{AUTHOR CONTRIBUTIONS}

RT-S, SM, and MK made substantial contributions to the conception and design of this project. RT-S obtained informed consent and clinical data. RT-S and MK wrote the manuscript. NM gave a genetic counseling perspective. SM and NM critically revised the manuscript. All authors read and approved the final manuscript.

\section{FUNDING}

This research reported in this publication was supported by the Strategic Health Innovation Partnerships Unit of the South African Medical Research Council, with funds received from the South African Department of Science and Technology (Research grant number S003665), and the Cancer Association of South Africa (CANSA). The South African BioDesign Initiative of the Department of Science and Technology and the Technology Innovation Agency is acknowledged for funding part of this research (grant number 401/01). DNA Genotek Inc., and Walter grant is acknowledged for sponsoring saliva kits used for sample collection. Dr. RS received a 2-year postdoctoral fellowship from Stellenbosch University. 


\section{ACKNOWLEDGMENTS}

Drs. Karin Baatjes and Jenny Edge are thanked for critical reading of this manuscript, and Nakita Laing for review of the informed consent description. Dr. Naftali Busakhala is acknowledged for return of actionable research results as the treating clinician. Dr. A. V. Peeters and K. E. Moremi are acknowledged for bioinformatics and research assistance. Profs. A. Zemlin and R. T. Erasmus are thanked

\section{REFERENCES}

Abeybsekera, T. (2018). Barriers to communication in the clinical consultation: how language barriers can affect consent. Independ. Student J Med. 1, 156-166.

Adebamowo, S. N., Francis, V., Tambo, E., Diallo, S. H., Landouré, G., Nembaware, V., et al. (2018). Implementation of genomics research in Africa: challenges and recommendations. Global Health Action 11:1419033. doi: 10.1080/16549716. 2017.1419033

Afolabi, M. O., Okebe, J. U., Mcgrath, N., Larson, H. J., Bojang, K., and Chandramohan, D. (2014). Informed consent comprehension in African research settings. Trop. Med. Int. Health 19, 625-642. doi: 10.1111/tmi.12288

Akuoko, C. P., Armah, E., Sarpong, T., Quansah, D. Y., Amankwaa, I., and Boateng, D. (2017). Barriers to early presentation and diagnosis of breast cancer among African women living in sub-Saharan Africa. PLoS One 12:e0171024. doi: 10. 1371/journal.pone.0171024

Alaei, M., Pourshams, A., Altaha, N., Goglani, G., and Jafari, E. (2013). Obtaining informed consent in an illiterate population. Middle East J. Digest. Dis. 5, 37-40.

Alfano, S. L. (2013). Conducting research with human subjects in international settings: ethical considerations. Yale J. Biol. Med. 86, 315-321.

Antal, H., Bunnell, H. T., McCahan, S. M., Pennington, C., Wysocki, T., and Blake, K. V. (2017). A cognitive approach for design of a multimedia informed consent video and website in pediatric research. J. Biomed. Informat. 66, 248-258. doi: 10.1016/j.jbi.2017.01.011

Appelbaum, P. S., Parens, E., Waldman, C. R., Klitzman, R., Fyer, A., Martinez, J., et al. (2014). Models of consent to return of incidental findings in genomic research. Hastings Cent. Rep. 6, 247-253. doi: 10.1111/j.1743-6109.2008. 01122.x

Baatjes, K. J. (2018). Bone Health in Post-menopausal Patients With Breast Cancer Treated With Aromatase Inhibitors: Factors Predicting the Risk for Osteoporosis, Ph.D. thesis, Stellenbosch University, Stellenbosch.

Baatjes, K. J., Conradie, M., Apffelstaedt, J. P., and Kotze, M. J. (2017). Pharmacogenetics of aromatase inhibitors in endocrine responsive breast cancer: lessons learnt from tamoxifen and CYP2D6 genotyping. Anti-Cancer Agents Med. Chem. 17, 1805-1813. doi: 10.2174/1871521409666170412124226

Bellcross, C. A., Lemke, A. A., Pape, L. S., Tess, A. L., and Meisner, L. T. (2009). Evaluation of a breast/ovarian cancer genetics referral screening tool in a mammography population. Genet. Med. 11, 783-789. doi: 10.1097/GIM. 0b013e3181b9b04a

Bhutta, Z. A. (2004). Beyond informed consent. Bul. World Health Organ. 82, 771-777. doi: 10.1590/S0042-96862004001000013

Boga, M., Davies, A., Kamuya, D., Kinyanjui, S. M., Kivaya, E., Kombe, F., et al. (2011). Strengthening the informed consent process in international health research through community engagement: the KEMRI-Wellcome Trust Research Programme experience. PLoS Med. 8:e1001089. doi: 10.1371/journal. pmed.1001089

Bombard, Y., Brothers, K. B., Fitzgerald-Butt, S., Garrison, N. A., Jamal, L., James, C. A., et al. (2019). The Responsibility to recontact research participants after reinterpretation of genetic and genomic research results. Am. J. Hum. Genet. 104, 578-595. doi: 10.1016/j.ajhg.2019.02.025

Busakhala, N. W., Chite, F. A., Wachira, J., Naanyu, V., Kisuya, J. W., Keter, A., et al. (2016). Screening by clinical breast examination in Western Kenya: who comes? J. Global Oncol. 2, 114-122. doi: 10.1200/jgo.2015. 000687

Busakhala, N., and Torrorey, R. (2012). "World breast cancer report," in Breast Cancer Situation of Western Kenya, (Lyon: iPRI Publication), 281-284. for critical reading and providing the infrastructure for this research. Prof. K. Moodley from the Stellenbosch University Centre for Medical Ethics and Law is acknowledged for the video (https://youtu.be/QjSfIA6_bfc) and pamphlets made available to assist with explanation of complex genetic terms during the feedback process. Any opinion, findings and conclusions or recommendations expressed in this article are those of the authors and the funders accept no liability in regard thereto.

Chen, H., Wu, J., Zhang, Z., Tang, Y., Li, X., Liu, S., et al. (2018). Association between BRCA status and triple-negative breast cancer: a meta-analysis. Front. Pharmacol. 9:909. doi: 10.3389/fphar.2018.00909

Christenhusz, G. M., Devriendt, K., and Dierickx, K. (2013). To tell or not to tell? A systematic review of ethical reflections on incidental findings arising in genetics contexts. Eur. J. Hum. Genet. 21, 248-255. doi: 10.1038/ejhg.2012.130

D’Andrea, E., Marzuillo, C., De Vito, C., Di Marco, M., Pitini, E., Vacchio, M. R., et al. (2016). Which BRCA genetic testing programs are ready for implementation in health care? A systematic review of economic evaluations. Genet. Med. 18, 1171-1180. doi: 10.1038/gim.2016.29

de Vries, J., Abayomi, A., Littler, K., Madden, E., McCurdy, S., Ouwe Missi OukemBoyer, O., et al. (2015). Addressing ethical issues in H3Africa research - the views of research ethics committee members. HUGO J. 9:1. doi: 10.1186/ s11568-015-0006-6

de Vries, J., Munung, S. N., Matimba, A., McCurdy, S., Ouwe Missi Oukem-Boyer, O., Staunton, C., et al. (2017). Regulation of genomic and biobanking research in Africa: a content analysis of ethics guidelines, policies and procedures from 22 African countries. BMC Med. Ethics 18:8. doi: 10.1186/s12910-016-0165-6

Dekking, S. A., van der Graaf, R., and van Delden, J. J. (2014). Strengths and weaknesses of guideline approaches to safeguard voluntary informed consent of patients within a dependent relationship. BMC Med. 12:52. doi: 10.1186/17417015-12-52

Emanuel, E. J. (2000). What Makes Clinical Research Ethical? JAMA: J. Am. Med. Ass. 283, 2701-2711. doi: 10.1001/jama.283.20.2701

Grant, K. A., Apffelstaedt, J. P., Wright, C., Myburgh, E., Pienaar, R., de Klerk, M., et al. (2013). Mammaprint pre-screen algorithm reduces chemotherapy in patients with early-stage breast cancer. S. Afr. Med. J. 103, 522-526. doi: 10.7196/SAMJ.7223

Grant, K. A., Myburgh, E. J., Murray, E., Pienaar, F. M., Kidd, M., Wright, C. A., et al. (2019). Reclassification of early stage breast cancer into treatment groups by combining the use of immunohistochemistry and microarray analysis. S. Afr. J. Sci. 115, 51-56. doi: 10.17159/sajs.2019/5461

Gupta, S., Nag, S., and Dawood, S. (2012). Challenges and opportunities in the design and implementation of breast cancer clinical trials in developing countries. Clin. Investig. 2, 579-587. doi: 10.4155/cli.12.46

Hill, J. A., Lee, S. Y., Njambi, L., Corson, T. W., and Dimaras, H. (2015). Cancer genetics education in a low- to middle-income country: evaluation of an interactive workshop for clinicians in Kenya. PLoS One 10:e0129852. doi: 10. 1371/journal.pone.0129852

Isles, A. F. (2013). Understood consent versus informed consent: a new paradigm for obtaining consent for pediatric research studies. Front. Pediatrics 1:38. doi: 10.3389/fped.2013.00038

Jarvik, G. P., Amendola, L. M., Berg, J. S., Brothers, K., Clayton, E. W., Chung, W., et al. (2014). Return of genomic results to research participants: the floor, the ceiling, and the choices in between. Am. J. Hum. Genet. 94, 818-826. doi: 10.1016/j.ajhg.2014.04.009

Jones, T., McCarthy, A. M., Kim, Y., and Armstrong, K. (2017). Predictors of BRCA1/2 genetic testing among Black women with breast cancer: a populationbased study. Cancer Med. 6, 1787-1798. doi: 10.1002/cam4.1120

Kamuya, D. M., Marsh, V. M., Njuguna, P., Munywoki, P., Parker, M., and Molyneux, S. (2014). "When they see us, it's like they have seen the benefits!": experiences of study benefits negotiations in community-based studies on the Kenyan Coast. BMC Med. Ethics 15:90. doi: 10.1186/1472-6939-15-90

Kamuya, D. M., Theobald, S. J., Marsh, V., Parker, M., Geissler, W. P., and Molyneux, S. C. (2015). The one who chases you away does not tell you go: 
silent refusals and complex power relations in research consent processes in coastal Kenya. PLoS One 10:e0126671. doi: 10.1371/journal.pone.0126671

Knoppers, B. M., Zawati, M. H., and Sénécal, K. (2015). Return of genetic testing results in the era of whole-genome sequencing. Nature Rev. Genet. 16, 553-559. doi: $10.1038 / \mathrm{nrg} 3960$

Kopanos, C., Tsiolkas, V., Kouris, A., Chapple, C. E., Albarca Aguilera, M., Meyer, R., et al. (2018). VarSome: the human genomic variant search engine. Bioinformatics 35, 1978-1980. doi: 10.1093/bioinformatics/bty897

Kotze, M. J. (2016). Application of advanced molecular technology in the diagnosis and management of genetic disorders in South Africa. S. Afr. Med. J. 106, S114-S118. doi: 10.7196/SAMJ.2016.v106i6.11012

Kotze, M. J., Lückhoff, H. K., Peeters, A. V., Baatjes, K., Schoeman, M., Van Der Merwe, L., et al. (2015). Genomic medicine and risk prediction across the disease spectrum. Crit. Rev. Clin. Lab. Sciences 52, 120-137. doi: 10.3109/ 10408363.2014.997930

Kotze, M. J., Schorn, D., and Coetzer, P. (2004). The impact of genetic testing on life insurance. J. Genomics Afr. Soc. 2004, 1-11.

Kotze, M. J., van Velden, D. P., Botha, K., Badenhorst, C. H., Avenant, H., van Rensburg, S. J., et al. (2013). Pathology-supported genetic testing directed at shared disease pathways for optimized health in later life. Personalized Med. 2013 10, 497-507. doi: 10.2217/pme.13.43

Lolkema, M. P., Gadellaa-van Hooijdonk, C. G., Bredenoord, A. L., Kapitein, P., Roach, N., Cuppen, E., et al. (2013). Ethical, legal, and counseling challenges surrounding the return of genetic results in oncology. J. Clin. Oncol. 31, 1842-1848. doi: 10.1200/JCO.2012.45.2789

Marshall, P. A. (2007). UNICEF/UNDP/World Bank/WHO special programme for research and training in tropical diseases., and World Health Organization. Ethical challenges in study design and informed consent for health research in resource-poor settings. Special Topics Soc. Econ. Behavioural Res. Report Series 5,79 .

Marshall, P. A., Adebamowo, C. A., Adeyemo, A. A., Ogundiran, T. O., Strenski, T., Zhou, J., et al. (2014). Voluntary participation and comprehension of informed consent in a genetic epidemiological study of breast cancer in Nigeria. BMC Med. Ethics 15:38. doi: 10.1186/1472-6939$15-38$

Ministry of Health Kenya (2013). National Guidelines for Cancer Management Kenya. Nairobi: Ministry of Health Kenya, 22.

Munung, N. S., Marshall, P., Campbell, M., Littler, K., Masiye, F., Ouwe-MissiOukem-Boyer, O., et al. (2016). Obtaining informed consent for genomics research in Africa: Analysis of H3Africa consent documents. J. Med. Ethics 42, 132-137. doi: 10.1136/medethics-2015-102796

Muthoni, A., and Miller, A. N. (2010). An exploration of rural and urban Kenyan women's knowledge and attitudes regarding breast cancer and breast cancer early detection measures. Health Care Women Int. 31, 801-816. doi: 10.1080/ 07399331003628453

Nembaware, V., Johnston, K., Diallo, A. A., Kotze, M. J., Matimba, A., Moodley, K., et al. (2019). A framework for tiered informed consent for health genomic research in Africa. Nature Genet. 51, 1566-1571. doi: 10.1038/s41588-0190520-x

Rayne, S., Schnippel, K., Firnhaber, C., Wright, K., Kruger, D., and Benn, C.-A. (2016). Fear of treatments surpasses demographic and socioeconomic factors in affecting patients with breast cancer in urban South Africa. J. Global Oncol. Online 22, 125-134. doi: 10.1200/JGO.2015.002691
Richards, S., Aziz, N., Bale, S., Bick, D., Das, S., Gastier-Foster, J., et al. (2015). Standards and guidelines for the interpretation of sequence variants: a joint consensus recommendation of the American College of medical genetics and genomics and the association for molecular pathology. Genet. Med. 17, 405-424. doi: 10.1038/gim. 2015.30

Sawe, R. T., Kerper, M., Badve, S., Li, J., Sandoval-Cooper, M., Xie, J., et al. (2016). Aggressive breast cancer in western Kenya has early onset, high proliferation, and immune cell infiltration. BMC Cancer 16:204. doi: 10.1186/s12885-0162204-6

Sawe, R. T., Mining, S. K., Ofulla, A. V., Patel, K., Guyah, B., Chumba, D., et al. (2017). Tumor infiltrating leukocyte density is independent of tumor grade and molecular subtype in aggressive breast cancer of Western Kenya. Trop. Med. Health 45, 1-11. doi: 10.1186/s41182-017-0059-4

Schoeman, M., Apffelstaedt, J. P., Baatjes, K., and Urban, M. (2013). Implementation of a breast cancer genetic service in South Africa - Lessons learned. S. Afr. Med. J. 103, 529-533. doi: 10.7196/SAMJ.6814

Silverman, H. J., Luce, J. M., Lanken, P. N., Morris, A. H., Harabin, A. L., Oldmixon, C. F., et al. (2005). Recommendations for informed consent forms for critical care clinical trials. Crit. Care Med. 33, 867-882. doi: 10.1097/01. CCM.0000159201.08203.10

Tindana, P., Bull, S., Amenga-Etego, L., de Vries, J., Aborigo, R., Koram, K., et al. (2012). Seeking consent to genetic and genomic research in a rural ghanaian setting: a qualitative study of the MalariaGEN experience. BMC Med. Ethics 13:15. doi: 10.1186/1472-6939-13-15

van der Merwe, N., Peeters, A. V., Pienaar, F. M., Bezuidenhout, J., Van Rensburg, S. J., and Kotze, M. J. (2017). Exome sequencing in a family with luminal-type breast cancer underpinned by variation in the methylation pathway. Int. J. Mol. Sci. 18:467. doi: 10.3390/ijms18020467

Van Der Merwe, N. (2018). Exome Sequencing in South Africa: Stakeholder Views on Feedback of Individual Research Results and Incidental Findings. M.Sc. thesis, University of Cape Town, Cape Town.

Wolf, S. M. (2013). Return of individual research results and incidental findings: facing the challenges of translational science. Annu. Rev. Genomics Hum. Genet. 14, 557-577. doi: 10.1146/annurev-genom-091212-153506

Wong, E. K., Bartels, K., Hathaway, J., Burns, C., Yeates, L., Semsarian, C., et al. (2019). Perceptions of genetic variant reclassification in patients with inherited cardiac disease. Eur. J. Hum. Genet 27, 1134-1142. doi: 10.1038/s41431-0190377-6

World medical Association (2013). WMA Declaration of Helsinki - Ethical Principles for Scientific Requirements and Research Protocols, (June 1964). France: World medical Association, 29-32.

Conflict of Interest: The authors declare that the research was conducted in the absence of any commercial or financial relationships that could be construed as a potential conflict of interest.

Copyright (®) 2020 Torrorey-Sawe, van der Merwe, Mining and Kotze. This is an open-access article distributed under the terms of the Creative Commons Attribution License (CC BY). The use, distribution or reproduction in other forums is permitted, provided the original author(s) and the copyright owner(s) are credited and that the original publication in this journal is cited, in accordance with accepted academic practice. No use, distribution or reproduction is permitted which does not comply with these terms. 